IdeAs

Idées d'Amériques

$18 \mid 2021$

Frontières dans les Amériques - Intégration, sécurité et migrations

\title{
Frontières dans les Amériques - Intégration, sécurité et migrations : approches critiques
}

Pierre-Alexandre Beylier, Anne-Laure Amilhat-Szary, Gregory Benedetti et Éric Tabuteau

\section{OpenEdition}

\section{Journals}

Édition électronique

URL : https://journals.openedition.org/ideas/11919

DOI : $10.4000 /$ ideas. 11919

ISSN : $1950-5701$

Éditeur

Institut des Amériques

Référence électronique

Pierre-Alexandre Beylier, Anne-Laure Amilhat-Szary, Gregory Benedetti et Éric Tabuteau, « Frontières dans les Amériques - Intégration, sécurité et migrations : approches critiques », IdeAs [En ligne], 18| 2021, mis en ligne le 01 octobre 2021, consulté le 21 octobre 2021. URL : http:// journals.openedition.org/ideas/11919 ; DOI : https://doi.org/10.4000/ideas.11919

Ce document a été généré automatiquement le 21 octobre 2021.

\section{(i) $\$ 9$

IdeAs - Idées d'Amériques est mis à disposition selon les termes de la licence Creative Commons Attribution - Pas d'Utilisation Commerciale - Pas de Modification 4.0 International. 


\section{Frontières dans les Amériques - Intégration, sécurité et migrations : approches critiques}

Pierre-Alexandre Beylier, Anne-Laure Amilhat-Szary, Gregory Benedetti et Éric Tabuteau

\section{Des frontières laboratoire}

1 Les Amériques constituent un laboratoire de premier ordre pour étudier les frontières et leurs évolutions. Non seulement, au cours de la décennie du «monde sans frontières ", le continent américain a multiplié les projets d'intégration régionale avec notamment le MERCOSUR et l'ALENA qui ont vu les pays se rapprocher et abaisser leurs barrières douanières mais, depuis quelques années, ces mêmes frontières sont devenues des points de crispation en raison d'un faisceau de problèmes qui se sont amplifiés - qu'il s'agisse de trafics en tout genre ou de tensions territoriales. Ces évolutions récentes nous amènent à envisager une lecture critique des processus frontaliers qui traversent les Amériques pour mieux les caractériser, à la fois dans leurs spécificités continentales et dans la manière dont cet ensemble rend lisibles les grandes tendances mondiales. Dans un contexte global de montée en théorie des études frontalières, il peut être intéressant de se demander en quoi une approche continentale permet de faire le point sur des spécificités régionales, mais aussi de contribuer, de façon originale, à cet effort épistémologique (Mezzadra S. et B. Neilson, 2013), (Nail T., 2016), (Parker N. et N. Vaughan-Williams, 2012), (Wastl-Walter D., 2012).

2 Ce numéro de la revue IdeAs souhaite en effet aborder la thématique des frontières par le prisme des deux dynamiques de prime abord antithétiques, de sécurisation et d'intégration, ainsi que de leur impact sur les migrations. En effet, prises dans des processus contradictoires de "démantèlement et de construction" (Machado De Oliveira T.-C., 2009: 19), non seulement ces dynamiques structurent des frontières désormais autant réticulaires que linéaires, servant à trier les flux de la mondialisation 
(Amilhat-Szary A.-L., 2015), mais elles se télescopent, ce qui, in fine, renforce l'ambivalence de l'institution frontalière.

3 Théâtre de l'appétit colonial des pouvoirs européens, le continent américain s'est retrouvé sous leur influence directe pendant près de quatre siècles, de sa (re)découverte en 1492 jusqu'à sa décolonisation dans la première moitié du XIX ${ }^{e}$ siècle. De l'établissement de zones d'influence à la création de véritables empires coloniaux, les Amériques sont devenues un prolongement de l'Ancien Monde et, source intarissable de ressources, une pièce maîtresse de l'économie-monde qui commençait à se développer. Depuis les décolonisations, elles constituent un espace où l'Occident réinvente constamment les modalités de son leadership global [Rouquié A., 1987].

Amériques ont donc cela de commun que leurs frontières ont été mises en place afin d'« ordonner » le continent (Popescu G., 2011), avant même la création d'Etatsnations. Plus précisément, ces frontières combinent de façon originale deux formes d'appropriation territoriale : d'une part, une logique de conquête zonale colonisatrice (frontier) qui vise à mettre en valeur un environnement considéré comme sauvage (wilderness) - une logique qui a été théorisée par Frederick Turner pour ce qui est des États-Unis (Turner F., 1964) - et, d'autre part, une volonté de maillage du monde dans une perspective occidentale de l'espace (boundary) (Perrier-Bruslé L., 2007).

5 Cette double logique de conquête s'est faite aux dépens des populations amérindiennes qui peuplaient le continent. Les pouvoirs coloniaux, puis les États nouvellement indépendants, se sont servis des frontières - système d'organisation de l'espace qui était étranger à ces populations autochtones - pour les asservir et les dominer en limitant leur mobilité, les assignant à résidence dans des réserves ou dans certains pays ou bien en leur imposant une identité «nationale » qui n'était pas la leur (Rensink B., 2018). La "violence » des frontières (Jones $R$, 2017) n'est donc pas nouvelle et, pour ce qui est des Amériques, leur caractère surimposé et exogène alors que d'aucuns les verraient comme antécédentes, ancre cette violence dans l'histoire même du continent, posant des questions en termes de légitimité et d'identité.

6 Au-delà de ces caractéristiques historiques qui leur sont propres, les frontières américaines partagent des traits communs avec les frontières du reste du monde. Dans un contexte plus large, marqué par l'essor de la mondialisation et les répercussions des attentats du 11 septembre 2001, elles sont aux prises de phénomènes a priori antithétiques et pourtant concomitants de debordering et de rebordering (Herzog L. et C. Sohn, 2019) : d'un côté, elles s'effacent, elles se défonctionnalisent pour faciliter le passage des flux commerciaux et financiers et, de l'autre, elles se raffermissent pour contrôler les mobilités de tous types, et pour stigmatiser certains types de migrations. Cela est caractéristique d'un phénomène qui différencie les modalités de franchissement d'une même frontière en fonction d'une combinaison de critères qui s'individualisent et définissent des « frontiérités » ou « borderities » disparates (Amilhat Szary, A.-L. et F. Giraut, 2015).

7 Leur apparente défonctionnalisation (Eva F., 1996) a pris son essor dans les années 1990, lorsque, embrassant les forces de la mondialisation, certains pays ont mis en place des accords commerciaux - l'ALENA pour l'Amérique du Nord ou le MERCOSUR pour l'Amérique du Sud, voire l'UNASUR à l'échelle hémisphérique. Ayant pour objectif de favoriser l'intégration régionale, ces accords ont mis en avant une vision particulière de la frontière, qui apparaissait davantage comme une « ressource » que comme un «stigmate » (Amilhat-Szary A.-L., 2015 : 85). L'intégration a été également 
envisagée comme un "facteur de stabilisation» (Medina L., 2009: 41) dans ce continent, dont certaines régions ont été marquées par des conflits frontaliers récurrents depuis le $\mathrm{xIX}^{\mathrm{e}}$ siècle et où les frontières sont, pour certaines, aujourd'hui encore contestées (Medina L., 2009 : 36-37).

Cependant, alors même qu'elles s'ouvraient au profit des échanges et de l'économie de marché, les frontières américaines ont vu certaines de leurs attributions se renforcer dans le même temps. Depuis les attentats du 11 septembre 2001, la menace du terrorisme international a poussé les pays du continent - États-Unis en tête - à repenser le rôle de leurs limites internationales. Afin de sécuriser les territoires, les frontières sont devenues le site de dispositifs de surveillance et de contrôle high-tech, pouvant converger dans l'érection de véritables "murs", symptomatiques de ce phénomène de refrontiérisation et de la violence qui lui est associée... Des frontières jusqu'ici quasiment non surveillées - à l'instar de la frontière Canada / États-Unis ou des dyades latinoaméricaines - ont vu se déployer toute une série de nouvelles mesures, des drones aux capteurs au sol, en passant par des tours équipées de caméras à vision nocturne (Beylier P.-A., 2016) . Si la lutte contre le terrorisme domine les discours, celle-ci semble être utilisée comme un prétexte pour contrôler les migrations qui sont désormais la cible explicite de ces mesures. La crise liée au COVID-19, qui a vu les frontières se verrouiller les unes après les autres, a conféré à ce phénomène une ampleur sans précédent, et le texte de Nicolli Bernardes Ribeiro aborde cette perspective aux frontières du Brésil, où se révèle la contradiction entre discours nationaliste et longue période de refus de la fermeture pour cause de pandémie.

C'est donc depuis cette perspective continentale que cette collection d'articles travaille la notion même de frontière, dont la définition problématique est désormais mise en avant. L'article de Jean Clot sur les "frontières poreuses" illustre l'adaptation des discours à ce contexte changeant. D'une manière complémentaire, François-Michel Le Tourneau met en évidence la « logique réticulaire » des orpailleurs en Guyane, lesquels profitent de l'absence de surveillance de ces zones frontalières reculées, ce qui va à contre-courant de la « logique aréale » des États pour lesquels les frontières demeurent des outils de souveraineté et de surveillance. Qu'elles soient contestées, transgressées, transcendées, renforcées ou intégrées, les frontières sont donc au cœur du débat politique dans les Amériques. Dans le débat public, elles semblent avoir pris le pas sur les discours promouvant l'intégration continentale au sein de l'ALENA et son successeur l'ACEUM, ainsi que du MERCOSUR, mais aussi à l'échelle de l'ensemble des deux Amériques (UNASUR), ensembles régionaux qui se présentaient comme des modèles dans les années 1990.

Cependant le «travail de la frontière » (pour traduire l'expression anglaise border work) n'est pas, dans les Amériques comme ailleurs, l'apanage des acteurs dominants : il est le fruit des pratiques " par le bas » (Rumford C., 2008) et les articles mettent l'accent sur la manière dont les habitants des régions frontalières interagissent avec les normes internationales qu'ils côtoient et défient, notamment dans le cas de l'orpaillage en Guyane qui semble s'émanciper de ces normes et fonctionner de manière " anationale », comme l'illustre le texte de François-Michel Le Tourneau. La frontière étant un "marqueur d'identité » (Piermay J.-L., 2005: 206), elle met en avant le questionnement sur le lien entre identité, territoire et frontière. La question est d'autant plus importante dans la mesure où, sur ce continent, "l'Etat a précédé la nation" (Medina L., 2009: 38). Le rôle assigné aux frontières dans la «cohésion 
nationale » (Ibid) apparaît donc d'autant plus important qu'il peut rentrer en conflit avec les représentations des communautés frontalières au sein desquelles l'interprétation que les populations autochtones font de la construction des frontières peut jouer un rôle essentiel (Nates Cruz B., 2013). Dans les textes réunis, ce sont souvent les négociations et les confrontations entre populations régionales et populations mobiles présentes dans les régions frontalières qui sont mises en avant, la question migratoire étant de plus conçue comme un problème central des espaces frontaliers américains aujourd'hui.

\section{Sécurité et migration aux frontières des Amériques, deux termes nécessairement liés?}

Les attentats du 11 septembre 2001 - et plus généralement l'émergence d'une menace terroriste internationale, présente en Amérique latine dès les attentats de Buenos Aires, dans les années 1980 - ont modifié le rôle des frontières. L'émergence d'une Fortress America (Alden E., 2008 ; Andreas P., 2003 ; Noble J., 2004) a été très largement documentée pour ce qui est des États-Unis mais le phénomène de rebordering concerne aussi les frontières latino-américaines. On lit ainsi sur ce continent une politique de réinterprétation originale des grandes tendances de gestion des frontières au niveau mondial, avec par exemple le déploiement d'un appareil de sécurisation des frontières brésiliennes d'une ampleur sans précédent, sans remise en question réelle de la croissance des flux d'échange internationaux, légaux comme illégaux (contrebande, narcotrafic, etc.) (Dorfman A. et al, 2014 ; Dorfman A. et al, 2017).

12 L'article de Beatriz Zepeda sur le travail des officiers de sécurité à la frontière guatelmalto-mexicaine ou le texte de Cléa Fortuné sur l'interprétation de la sécurité que fait la communauté transfrontalière de Douglas (Arizona) / Agua Prieta (Sonora) donnent ainsi une vision nuancée du travail humain et des négociations qui, au quotidien, construisent le grand paradigme de la « sécurité ». On s'intéresse ainsi aux aspects matériels d'une telle dynamique, et à la façon dont ce processus se territorialise. Les approches historiques qui renouvellent la question du conflit frontalier territorialisé et multiplient les échelles de lecture, permettent, dans ce contexte, de faire évoluer les récits nationaux et nationalistes.

13 Les différentes politiques mises en place depuis le début des années 2000 dans les Amériques fournissent une illustration particulière du phénomène de rebordering qui est en jeu à l'échelle mondiale. Les articles réunis ici travaillent à la fois les dispositifs et leurs implications pour les relations transfrontalières, dans une approche comparative (cf. articles de Jean Clot ou Laurence Cros), qui permet non seulement de mettre en évidence les dynamiques qui traversent l'Amérique du Nord, mais aussi de lier les deux moitiés des Amériques... (Brunet-Jailly E., 2007; Konrad V et H. Nicol, 2008).

Malgré les phénomènes communs qui les structurent, les frontières américaines véhiculent chacune une certaine spécificité puisque leur rôle change d'un pays à l'autre, voire d'une région à l'autre (Machado de Oliveira T.-C., 2009:20) : elles sont marquées, au contraire, par une " immense variété » notamment en Amérique latine où elles sont plus nombreuses. Entre les «frontières distantes » qui séparent des régions marginales dont les territoires «tournent le dos à la frontière " (Argentine / Chili, 
Paraguay / Brésil...), les «frontières capricantes », marquées par des liens transfrontaliers illégaux notamment dans des zones nouvellement urbanisées (Costa Rica / Nicaragua, Mexique / Guatemala), ou encore les "frontières vibrantes ", qui tirent leur dynamisme d'une population dense et d'avantages comparatifs nombreux (Brésil / Uruguay, Pérou / Equateur, Mexique / Etats-Unis), sans oublier les « frontières protocolaires ", qui sont des régions instrumentalisées par le pouvoir central afin de promouvoir leur «dynamisation » ou encore de lutter contre les trafics illégaux selon une approche top-down (Chili / Argentine, Haïti / République dominicaine), on voit que les types de frontières sont nombreux (Machado de Oliveira T.-C., 2009: 28-30). Différents degrés de coopération transfrontalière se nouent à travers elles, donnant lieu à une montée en puissance de relations inter-territoriales dites paradiplomatiques (Gonzalez Miranda, S. et al., 2016).

Les traversées des frontières et leur coût humain croissant apparaissent aujourd'hui comme des éléments essentiels de la compréhension des frontières américaines (De Leon J. et M. Wells, 2015). De façon saisissante, différents articles du dossier (ceux de Marie-Laure Coubès ou Jean Clot) montrent combien le passage de la ligne ne peut plus se comprendre que de manière pluriscalaire. Il faut en effet analyser à la fois les flux intracontinentaux, liés aux mobilités de travail notamment, mais aussi la façon dont les Amériques s'inscrivent dans des stratégies migratoires de grande ampleur, avec des personnes de plus en plus nombreuses qui, par exemple, tentent d'arriver en Amérique du Nord depuis l'Afrique, en traversant l'Atlantique par la vieille route des esclaves puis, en tentant leur chance sur des itinéraires de remontée vers le nord longs et périlleux. Cela met en avant les questions de vulnérabilité et de risque, et les stratégies mises en œuvre par les communautés frontalières pour vivre avec l'insécurité (cf. le texte de Cléa Fortuné), et réinterpréter à leur manière les injonctions internationales. Les phénomènes illégaux qui se sont développés dans les Amériques (le narcotrafic, l'immigration illégale ou bien les cartels, etc.) ne peuvent être abordés sans creuser leurs causes, leurs ramifications, leurs implications pour les populations locales ainsi que les politiques mises en place pour les combattre. Ils ne doivent pas faire oublier les multiples manières dont les populations font avec la frontière, au quotidien, pour l'éducation ou la santé notamment (cf. les articles d'Elisabeth Fauquert ou Isabelle Sinic).

16 Enfin, les espaces frontaliers étant des lieux en perpétuelle évolution dont l'expression esthétique et les imaginaires se recomposent rapidement (Rodney L., 2017 ; AmilhatSzary A.-L., 2014), la médiation artistique de l'imaginaire de la ligne apparaît comme un point d'entrée essentiel pour comprendre l'évolution en cours des frontières américaines. Le texte de Marion Gautreau consacré à l'approche photographique de la frontière Mexique / États-Unis, analysée de manière rétrospective sur plus de quarante ans à travers le travail d'Elsa Medina et de Francisco Mata Rosa, répond à cette quête.

\section{La logique de l'organisation du numéro}

Les articles présentés ici déclinent de trois manières complémentaires la problématique commune. Trois d'entre eux travaillent la définition de la sécurité, pour montrer son aspect à la fois multiforme et multiscalaire d'une part, et l'importance de l'interprétation que les acteurs locaux font des enjeux globaux. Il s'agit de l'article de Beatriz Zepeda intitulé «Les limites du pouvoir. La configuration géopolitique des 
frontières du Mexique au xix ${ }^{e}$ siècle ", de celui de François-Michel Le Tourneau, "La frontière ? Quelle frontière ? La dynamique transnationale de l'orpaillage clandestin en Guyane française" ainsi que du texte de Nicolli Bernardes Ribeiro, "Contingence pandémique et impacts migratoires : une analyse des actes normatifs pour fermer la frontière entre le Brésil et le Venezuela ».

Une deuxième série de textes s'intéresse à la question migratoire, pour interroger son lien complexe aux problématiques de sécurité. Il s'agit des articles de Marie-Laure Coubès, «De travailleurs mexicains sans-papier à familles centraméricaines demandeuses d'asile : la transformation des flux migratoires à la frontière MexiqueÉtats-Unis, 2005-2020)", de celui de Laurence Cros, "Demandes d'asile à la frontière américano-canadienne : l'Entente entre le Canada et les États-Unis sur les tiers pays sûrs et les valeurs canadiennes" et enfin de Jean Clot sur "La frontière entre le Mexique et le Guatemala : une frontière "poreuse" ?».

Le dernier groupe d'articles nuance de manière stimulante cette opposition souvent caricaturale entre sécurité et migration, montrant comment celle-ci est négociée au quotidien par des pratiques qui en renouvellent les termes. Ce que démontre Cléa Fortuné de manière globale à l'échelle d'une agglomération dans «Tensions dans la sécurité frontalière : entre sécurité étatique et insécurité économique locale » apparaitt à travers les usages scolaires dans le texte d'Isabelle Sinic, "Coopération éducative entre les États-Unis et le Mexique: quelles perspectives pour les élèves transnationaux?») ou médicaux de la frontière à travers celui d'Elisabeth Fauquert, "Sometimes the treatment you seek is just one flight away" : causes et limites des nouvelles mobilités de santé étatsuniennes au XxI ${ }^{\mathrm{e}}$ siècle »). Enfin, dans « Regards sur la ligne. La frontière Mexique - États-Unis à travers l'objectif d'Elsa Medina et de Francisco Mata Rosas » Marion Gautreau nous offre un texte dont l'approche historique confirme les analyses contemporaines et dont la dimension artistique les prolonge.

\section{Ouvertures}

20 Au-delà du tour d'horizon proposé par la collection d'articles recueillie pour ce numéro, un certain nombre de thèmes restent à approfondir pour mieux comprendre l'évolution des frontières américaines. La question des frontières maritimes dont la délimitation pose de vives tensions, notamment en Amérique centrale (Medina L., 2009: 40 et Medina L., 2021), demanderait ainsi à être mieux documentée sur le continent. Elle ouvre ainsi la réflexion sur les frontières externes du continent, notamment sur le front arctique (Nicol H et L. Heininen, 2009). Elle interroge enfin, de façon plus générique, la dimension environnementale des questions de frontière (Guyot S., 2011; Wadewitz L., 2012), qui prend une dimension singulière dans les Amériques où, pour une majorité, les limites internationales traversent des zones de faible densité d'occupation humaine.

21 Il faut enfin souligner que le continent américain reste strié par une myriade de frontières autres que géopolitiques: sociales et raciales notamment. La question spécifique des frontières urbaines (Chevalier J. et C. Carballo, 2004) dans des espaces continentaux qui partagent la même expérience d'un développement lié au néolibéralisme économique, pose en effet de nombreuses questions concernant leur 
segmentation. Des approches intersectionnelles restent à construire pour continuer à explorer la multi dimensionnalité des processus frontaliers dans les Amériques.

\section{BIBLIOGRAPHIE}

Alden, Edward, The Closing of the American Border, New York City, Harper and Collins, 2008.

Andreas, Peter et Thomas J. Biersteker, The Rebordering of North America: Integration and Exclusion in a New Security Context, New York, Routledge, 2003.

Amilhat-Szary, Anne-Laure, « Latin American Borders on the Lookout: Recreating borders through art in the Mercosul ", Making the Border in Everyday Life, dir. Jones, Reece et Corey Jonhson, Farnham, Ashgate, 2014, p. 346-78.XAmilhat Szary, Anne-Laure, Qu'est-ce qu'une frontière aujourd'hui ? Paris, PUF, 2015.

Amilhat Szary, Anne Laure, et Frédéric Giraut (dir.), Borderities: The Politics of Contemporary Mobile Borders, Basingstoke, Palgrave Macmillan, 2015.

Beylier, Pierre-Alexandre, Canada-États-Unis, les enjeux d'une frontière, Rennes, Presses universitaires de Rennes, coll. Des Amériques, 2016

Brunet-Jailly, Emmanuel (dir.), Borderlands: Comparing Border Security in North America and Europe, Ottawa, University of Ottawa Press, 2007.

Chevalier, Jacques et Cristina Carballo, « Fermetures résidentielles et quête de l'entre-soi, entre Nord et Sud Des Amériques ", L'espace Géographique, vol. 33, n 4, 2004, p. 325-335.

De Leon, Jason, et Michael Wells, The Land of Open Graves, Los Angeles, University of California Press, 2015.

Dorfman Adriana, et Daniel Francisco de Bem, « Contrabando, Tragédia e Reflexividade: Antígona Na Fronteira Gaúcha », in Revista Do Centro de Educação e Letras, n 15, 2014, p. 33-51.

Dorfman, Adriana et al., « Political Commodities and Sovereignty Management: Cigarette Smuggling across Brazil's Southern Borders », in Geopolitics, vol. 22, n 4, 2017, p. 863-886 <https://doi.org/10.1080/14650045.2017.1356288> page consultée le 13 octobre 2021.

Eva, Fabrizio, 1996, « International Boundaries, Geopolitics and the (Post)Modern Territorial Discourse: The Functional Fiction ». in Newman, David (dir.), Boundaries, Territory and Postmodernity, London, Taylor \& Francis, p. 32-51.

Gonzalez Miranda, Sergio, et al. (dir.), Relaciones transfronterizas y paradiplomacia en América Latina. Aspectos teóricos y estudio de casos, Santiago de Chile, RIL editores, 2016.

Guyot, Sylvain, « The Eco-Frontier Paradigm: Rethinking the Links between Space, Nature and Politics » Geopolitics vol. 16, n³ 2, 2011, p.675-706.

Herzog, Lawrence A., et Christophe Sohn, « The Co-Mingling of Bordering Dynamics in the San Diego-Tijuana Cross-Border Metropolis » Territory, Politics, Governance vol. 7, n² 2, 2019, p. 177-99, https://doi.org/10.1080/21622671.2017.1323003, page consultée le 13 octobre 2021.

Jones, Reece, Violent Borders: Refugees and the Right to Move, London / New York, Verso, 2017. 
Konrad, Victor, et Heather Nicol, Beyond Walls: Re-Inventing the Canada-United States Borderlands, London, Ashgate, 2008.

Machado de Oliveira, Tito Carlos, «Frontières en Amérique latine : réflexions méthodologiques », Espaces et sociétés, vol. 138, n³, 2009, p. 19-33.

Medina, Lucile, «Les frontières de l'isthme centraméricain, de marges symboliques à des espaces en construction ", Espaces et sociétés vol. 138, n³, 2009, p. 35-50.

Medina, Lucile, « Pouvoir, préservation, prédation. Les frontières d'Amérique latine témoins d'un continent sous tensions » Revue L'Espace Politique. Revue en ligne de géographie politique et de géopolitique, $\mathrm{n}^{\circ}$ 42, 2021, doi: 10.4000/espacepolitique.9424, page consultée le 13 octobre 2021

Mezzadra, Sandro, et Brett Neilson, Border As Method, or, the Multiplication of Labor, Durham, NC, Duke University Press, 2013.

Nail, Thomas, Theory of the Border, Oxford / New York, Oxford University Press, 2016.

Nates Cruz, Beatriz (dir.), La Frontera, Las Fronteras. Diálogos Transversales En Estudios Territoriales Contemporáneo, Editorial Universitaria de Caldas, 2013.

Nicol, Heather et Lassi Heininen, « Networking the North: Cross Border Connections and the New International Circumpolar Geopolitics » in Southern Journal of Canadian Studies, vol. 2009/2, 2009, p. 11-26.

Noble, John, « Fortress America or Fortress North America? », paper prepared for the IRPP on North American Integration: Migration, Trade and Security, April 2004.

Parker, Noel et Nick Vaughan-Williams, « Critical Border Studies: Broadening and Deepening the "Lines in the Sand" Agenda » in Geopolitics, vol. 17, 2012, p.727-33.

Perrier-Bruslé, Laetitia, « The Front and the Line: The Paradox of South American Frontiers Applied to the Bolivian Case » in Geopolitics, vol.12, 2007, p. 57-77.

Piermay, Jean-Luc, «La Frontière et ses ressources : regards croisés » in Antheaume, Benoît et Frédéric Giraut (dir.) Le territoire est mort : vive les territoires ! : une refabrication au nom du développement, Montpellier, IRD Editions, 2005, p. 2013-221.

Popescu, Gabriel, Bordering and Ordering the Twenty-first Century: Understanding Borders, Plymouth, Rowman and Littlefield Publishers Inc., 2011.

Rensink, Brenden, Native but Foreign, Indigenous Immigrants and Refugees in the North American Borderlands, College Station, TX, Texas A\&M University Press, 2018.

Rodney, Lee, Looking beyond Borderlines: North America's Frontier Imagination, New York, Routledge, Taylor \& Francis Group, Routledge Advances in Art and Visual Studies n¹9, 2017.

Rouquié, Alain, Amérique latine : introduction à l'Extrême-Occident, Paris, Seuil, 1987.

Rumford, Chris (dir.), Citizens and Borderwork in Contemporary Europe, New York / London, Routledge, 2008.

Turner, Frederick. J., La Frontière dans l'histoire des États-Unis, Paris, Presses universitaires de France, 1963 [1893].

Wadewitz Lissa K., The Nature of Borders. Salmon, Boundaries, and Bandits on the Salish Sea, Bellingham, University of Washington Press, 2012.

Wastl-Walter, Doris (dir.), Companion to Border Studies, Farnham, Ashgate, 2012. 


\section{AUTEURS}

\section{PIERRE-ALEXANDRE BEYLIER}

Pierre-Alexandre Beylier est actuellement maître de conférences en études nord-américaines à l'université Grenoble-Alpes. Ancien élève de l'Ecole Normale Supérieure de Cachan et Agrégé d'anglais, il a effectué une thèse à l'Université Paris 3-Sorbonne Nouvelle, portant sur les mutations qu'a connues la frontière Canada/Etats-Unis depuis le 11 septembre 2001. Il a publié aux Presses Universitaires de Rennes un ouvrage sur ce sujet : Canada/Etats-Unis : les enjeux d'une frontière. Il s'intéresse désormais aux questions relatives aux communautés frontalières et aux villes-frontière.

\section{ANNE-LAURE AMILHAT-SZARY}

Anne-Laure Amilhat Szary est professeure à l'Université Grenoble-Alpes. Ancienne élève de l'ENS Fontenay, agrégée de Géographie et membre honoraire de l'IUF, c'est une géographe politique qui analyse les frontières. Elle développe des recherches concernent les interrelations entre espace et art dans les lieux contestés et construit un musée imaginaire du border art. Cofondatrice du collectif antiAtlas des frontières (http://www.antiatlas.net/), elle anime le Performance Lab dédié à la Recherche-Création.

Auteure de : Après les frontières, avec la frontière (2006, avec M.-C. Fourny), Qu'est-ce qu'une frontière aujourd'hui ? (2015), Borderities, the Politics of Mobile Borders (2015, avec F. Giraut), Histoires de frontières, une enquête sud-africaine (2017, collectif), Géopolitique des frontières. Découper la terre, inventer une vision du monde (2020), Frontières (2020, avec G. Hamez)

\section{GREGORY BENEDETTI}

Gregory Benedetti, agrégé d'anglais et docteur en civilisation américaine, est maître de conférences à l'Université Grenoble Alpes, et membre de l'Institut des langues et des cultures d'Europe, Amérique, Afrique, Asie et Australie. Ses recherches portent essentiellement sur la question raciale aux États-Unis, et plus particulièrement sur la communauté afro-américaine, tant sur le plan politique que culturel. Ses premières publications s'intéressaient notamment à la nouvelle génération d'hommes politiques noirs, dont Barack Obama est devenue la figure tutélaire, mais non exclusive, en devenant président des États-Unis en 2008. Ses travaux s'intéressent également à l'histoire et la politique afro-américaine dans son ensemble, ainsi qu'à la représentation de la communauté noire dans les productions culturelles comme les séries télévisées. Il a dernièrement travaillé sur les élections présidentielles américaines en coorganisant une journée d'études à l'Université Grenoble Alpes. Il est membre de l'Association française d'études américaines depuis 2008.

\section{ÉRIC TABUTEAU}

Professeur des Universités, Université d'Orléans.

UFR des lettres, langues et arts. 